\title{
EL NACIMIENTO DE LA ÚLTIMA AUDIENCIA INDIANA. SEDE, ARTISTAS Y COSTES DE LA AUDIENCIA DEL CUZCO
}

\author{
POR MARÍA JESÚS MEJÍAS
}

\begin{abstract}
Tras una breve alusión a los problemas habidos para la creación de la Audiencia de Cuzco, pasamos al análisis y descripción de su sede, de sus obras de acondicionamiento y de los artistas y artesanos que rabajan en ella, para terminar con la relación de gastos efectuados. Aportamos, además, documentación procedente del A.G.I., que nos describe el estado de la casa de la Audiencia tras sus obras de remodelación.

After a short allusion to the problems related to the creation of the Audiencie of Cuzco, we analyze and make a description of its site, of its works if conditioning, and the artits and craftomen who took part in it. We also include the list of the undertaken expenses. Besides that we add the documents proceeding from the A.G.I. which desribe the state of the Audiencie's building after its reconstructing.
\end{abstract}

La audiencia castellana nacida en la Baja Edad Media y estructurada definitivamente en la Edad Moderna, compuesta por un número variable de jueces letrados, llamados oidores para lo civil y alcaldes del crimen para lo criminal, y presidida por un gobernador o regente, es la que se trasplantó a América adquiriendo un gran desarrollo. Su papel era muy importante, tanto en el orden judicial (ribunales de apelación) como en lo gubernativo, ya que a ellas pasaron numerosas facultades que correspondían al Consejo de Indias, al que no se podía recurrir con facilidad a causa de la distancia ${ }^{1}$.

En América, la primera Audiencia se instaló en 1511 en Santo Domingo. Posteriormente fueron multiplicándose, creándose en el siglo XVI la mayoría de ellas (México,

1. Existen gran cantidad de estudios dedicados al análisis de las audiencias indianas, sólo reseñaremos los que nos parecen claves: Ruiz Guiñazu,E.: La Magistratura Indiana. 1916; Pelsmaeker, F.: La Audiencia en las colonias españolas de América, 1926; García Gallo,A.:Estudios de H $^{\mathbf{a}}$ de Derecho Indiano, 1972; Las Audiencias Indianas.Su origen y caracteres, 1975; Suárez,S.G.: Las Reales Audiencias indianas:fuentes y bibliografia, 1989. 
1527/1531; Panamá, 1535; Lima, 1542; Guatemala, 1543; Guadalajara, 1548; Santa $\mathrm{Fe}, 1549$; Charcas, 1559; Quito, 1563). En el siglo XVII nacen las de Santiago de Chile en 1609 y la de Buenos Aires en 1661, y, por último en el siglo XVIII, en plenas reformas borbónicas, se crea la audiencia de Caracas en 1786, y en 1787 la última audiencia indiana, la del $\mathrm{Cuzco}^{2}$.

Aunque no es nuestro propósito hacer un estudio, ni siquiera parcial, de lo que significó en el plano judicial la Audiencia cuzqueña, debemos aludir a los problemas habidos para su creación, y al entorno político-social en la que surge. Con ello comprenderemos mejor la elección de su sede y los costes de su acondicionamiento.

\section{LA CREACIÓN DE LA AUDIENCIA DE CUZCO}

Un Real Decreto expedido por Carlos III el 26 de febrero de 1787 y ratificado por Real Cédula fechada en Aranjuez a 3 de mayo de $1787^{3}$, disponen la creación y establecimiento de la Audiencia de Cuzco. Su inaguración no tuvo lugar hasta el 3 de noviembre de 1788, y fue acompañada de los oportunos festejos y solemnidades. De éstos queda constancia en la "Relación de la fundación de la Real Audiencia del Cusco", obra encargada por el regente de la Audiencia, José de la Portilla y Gálvez, al presbítero Ignacio de Castro ${ }^{4}$.

Para muchos, los desazones sociales, políticos y económicos mejorarían con la existencia de una Audiencia cercana que remedíara la sensación de olvido y abandono judicial, que experimentaba la ciudad del Cuzco. Recordemos que la situación geográfica de esta ciudad no permite la fácil comunicación con las ciudades de Lima o de Charcas, en las que se encontraban las Audiencias más próximas.

Si bien la Audiencia se crea a fines del siglo XVIII, la necesidad de su existencia venía manifestándose desde principios del siglo XVII. Así, lo declaran tanto Juan de Solórzano Pereira en su Política Indiana (1647), libro V, capítulo $3^{\circ}$, como Túpac Amaru en su carta al visitador José Antonio de Areche, fechada el 5 de marzo de $1781^{5}$. Sin duda, la revolución que dirige Túpac Amaru exigiendo

2. La bibliografía existente sobre la Audiencia de Cuzco es más bien escasa. Las obras de carácter general la tratan de pasada, y los estudios específicos son pocos y de diversa consideración. Reseñaremos los tres que nos parecen esenciales: VALCARCEL,C.D.: Documentos de la Audiencia de Cusco en el A.G.I. Lima 1957; TORERO GOMERO,Carmen: Establecimiento de la Audiencia del Cuzco. Boletín del Instituto Riva-Agüero, $n^{\circ}$ 8, Lima, 1969-1971. Páginas 374-522. VARCALCEL, C.D.: La Audiencia del Cuzco. Memoria del II Congreso Venezolano de Historia. Tomo III, Caracas 1975. Páginas 291-297.

3. A.G.I. Cuzco 4. Real Cédula de fundación de la Audiencia de Cuzco.

4. CASTRO, Ignacio de: "Relación de la Fundación de la real Audiencia del Cuzco en 1788 y de las Fiestas con que esta grande y fidelísima ciudad celebró este Honor". Obra póstuma editada por el eclesiástico Sebastián de la Paliza, e impresa en Madrid en 1795.

En el A.G.I., Cuzco 21, expediente $n^{\circ} 8$, podemos ver la documentación relacionada con la solicitud que hace la ciudad de Cuzco pidiendo permiso para imprimir la Relación escrita por Castro.

5. VALCARCEL,C.D.: La Audiencia del Cuzco. Memoria del II Congreso Venezolano de Historia. Tomo III, Caracas 1975. Páginas 291-297. 
reformas político-sociales, conlleva la revindicación de la creación de una Audiencia en Cuzco como medida de reforma judicial. De hecho podemos considerar su creación como una de las consecuencias de esta revolución.

\section{LA SEDE DE LA AUDIENCIA}

La situación económica en el último cuarto del siglo XVIII, no estaba para lujos ni gastos excesivos, por eso la sede de la nueva audiencia debía compaginar las mejores condiciones posibles con el más austero de los gastos.

Sometidos a esta presión económica se pensó reutilizar un edificio de la ciudad. En principio tres eran los edificios candidatos: el edificio de la Compañía de Jesús, expropiado y utilizado como cuartel, en la Plaza Mayor; la casa de la Moneda situada en la Plaza del Regocijo, y el edificio del Cabildo de la ciudad, también en la plaza del Regocijo.

La elección se polarizó entre los partidarios de la Casa del Cabildo y los del edificio de la Compañía. Los grandes gastos que suponía rehabilitar el edificio de la Compañía de Jesús, así como los informes favorables a la Casa del Cabildo dados tanto por el cesante intendente, Benito de la Mata Linares, y el teniente asesor de la intendencia, Ignacio Delgado, determinaron que el regente de la Real Audiencia, José Portilla y Gálvez, se decidiera por la Casa del Cabildo, considerada más austera en los gastos de su reparación ${ }^{6}$.

El primer informe que recibe el regente de la Audiencia, José Portilla, es el que le envía Mata Linares. En él se mostraba claramente partidario de tener como sede de la Audencia la Casa del Ayuntamiento, y para reforzar su opinión ajunta al informe un plano ${ }^{7}$ (figura 1) sugeriendo la futura distribución de ésta. Sus argumentos se basan exclusivamente en la moderación de los gastos de acondicionamiento, que calculaba no pasarían de los 4.000 pesos.

Los partidarios de colegio de la Compañía de Jesús se hacían cada vez más fuertes, y pretendían establecer el Tribunal en el primer claustro del citado Colegio. Consideraban que el coste de la adecuación del edificio sería sólo un poco mayor que el de la casa del Ayuntamiento, pero que se compensaría con la calidad de la sede, más cómoda, amplia y digna.

A la vista de todo esto, Portilla solicita un informe sobre el estado y condiciones del citado edificio. El encargado de realizarlo es en esta ocasión el teniente asesor, Ignacio Delgado, claramente partidario de la Casa del Cabildo, a la vista de las informaciones que realiza. Este le envia una completa relación, acompañada de plano ${ }^{8}$ (figura 2),y fechada a 1 de enero de 1788 , en la que

6. TORERO GOMERO,C.: Establecimiento de la Audiencia del Cuzco. Boletín del Instituto RivaAguiero, $n^{\circ} 8$, Lima, 1969-1971. Páginas 441 a 447.

7. A.G.I. Mapas y Planos, Perú y Chile, 268, 268bis.

8. A.G.I. Mapas y Planos, Perú y Chile, 267, 267bis. 
resaltaba los inconvenientes que el edificio ofrecía para tal función ${ }^{9}$ Entre estos destacaba: la distancia a la que se encontraba de la plaza principal, o del Regocijo; la ocupación que de él hacían los cuerpos de Infantería, Artillería y Caballería, y las oficinas de la Aduana y Correos; y sobre todo, los grandes costes que supondrían su acondicionamiento.

En la sesión del 30 de junio de 1788, el Cabildo de la ciudad transfiere parte de su casa para la sede de la Audiencia sin presentar oposición alguna. La única condición que el Cabildo establece es que en el mismo edificio quedara fijada su Sala Capitular "con las mismas proporciones y desencia que ahora, y corresponde a este mismo cuerpo: Lo que oydo por los señores concurrentes, todos uniformemente dixeron que no hallaron el menor reparo para dicha cesion" ${ }^{10}$.

El acuerdo de cesión funcionó hasta 1814, año en el que el Cabildo reclama las habitaciones cedidas para acomodar en ellas a la Diputación Provincial. Esta circunstancia es aprovechada por la Audiencia para solicitar ante la Corona una sede propia que se ajuste "con decoro y decencia" a su representación, pues las 45 varas que ocupaba ya no eran suficientes. Por lo tanto, se vuelve a considerar las propuestas anteriores de la Casa de la Moneda y la del edificio de la Compañía de Jesús, insistiendo en esta última ${ }^{11}$. El tiempo parece haberles dado la razón a los primeros partidarios del Colegio de los Jesuítas que demostraron tener mayor visión de futuro.

\section{2.a. Obras y acondicionamiento .}

Acordada la cesión, la sede de la Nueva Audiencia se establecería en los altos de la casa del Cabildo, pero para ello antes había que acometer varias obras de acondicionamiento. Se nombró a Andrés Graz como comisionado para la dirección de las obras, y como alarifes a José Palomino y a Martín Bustamante, aunque todo estaría supervisado por el regente Portilla.

La obra necesitó de casi un año para su terminación, ya que el acondicionamiento de la casa suponía una auténtica transformación de ésta. En la planta baja se dispuso la nueva Sala Capitular del Cabildo, para lo que hubo que restructurar el corral y un corredor. En la planta alta, sede real de la Audiencia, se proyectaron la

9. A.G.I. Cuzco 21 y Lima 697." Testimonio del expediente promovido sobre la habilitación de la Casa y compra de muebles necesarios para la formación de la Real Audiencia del Cuzco y sus oficinas", 30 folios. Documento anejo a la carta del virrey de Perú, Francisco Gil, fechada en Lima a 16 de marzo de 1791, en la que solicita la aprobación de los gastos habidos en la casa y muebles de la Audiencia.

10. A.G.I. Cuzco, 21. Copia del Acta del Cabildo fechada en Cuzco a 30 de junio de 1788, incluida en el testimonio sobre los gastos de las obras de la casa de la Audiencia,

anejo a una carta del virrey del Perú, Francisco Gil, fechada en Lima a 16 de marzo de 1791 en la que solicita la aprobación de los gastos.

11. A.G.I. Cuzco, 8. Carta de la Audiencia del Cuzco, fechada en Cuzco a 26 de mayo de 1814, informando sobre la precariedad de su sede y de la necesidad de trasladarse de edificio. Le acompaña testimonio con informes de las deficiencias de la Casa, y de los edificios considerados como posibles alternativas, deteniéndose especialmente en el de la Compañía de Jesús. 
sala de procuradores, la sala de Justicia, la sala de Acuerdo y el Oratorio. Para ello hubo que rehacer la escalera principal de la casa, levantar nuevas paredes, restaurar la balconada y corredor que daba al patio, hacer suelos nuevos, abrir ventanas y puertas nuevas, y construir unos arcos grandes para la portada.

La distribución de la Casa ${ }^{12}$ al terminar la obra fue la siguiente: en la planta baja, a la derecha del zaguán, se encontraba la escribanía de Cámara, y a la izquierda la Chancillería Real; seguidamente, el patio distribuía unas dependencias de uso del gobernador intendente y la Sala Capitular para uso del Cabildo, y al fondo se establecieron el corral y la caballeriza, así como la cárcel. En la planta superior, subiendo a la derecha, se encontraba una capilla dedicada a Santa Bárbara, y al final de la escalera, en un corredor, se distribuían la sala de los procuradores, la de Justicia, la de Acuerdo y el oratorio ${ }^{13}$.

\section{2.b. Artistas y artesanos que trabajan en las obras.}

Según las indicaciones del regente Portilla, para mayor moderación de los gastos, los artistas y artesanos que trabajaran en la obra y acondicionamiento de la Casa del Cabildo debían ser locales, y los materiales comprados en la misma ciudad.

En primer lugar, como ya hemos indicado, se designaron a dos alarifes vecinos de la ciudad del Cuzco, José Palomino y Martín Bustamante, "unicos facultativos que entienden y dirigen obras de este genero en aquella ciudad" ${ }^{14}$.

En el testimonio sobre los gastos habidos en la adecuación de la Casa del Cabildo ${ }^{15}$, se hace mención de pagos a varios herreros, carpinteros, pintores, bordadores, hojalatero, doradores, fundidor y relojero que trabajaron en ella; esto nos demuestra que las obras realizadas fueron de cierta envergadura, a pesar de los deseos de economizar. El documento no es muy explícito a la hora de describirnos los trabajos de los artistas. En la mayoría de las anotaciones, sólo se hace referencia al dinero pagado "por las hechuras de todas las obras que trabajó" el artista en cuestión; aún así, es de gran interés la relación de artistas, vecinos de la ciudad del Cuzco, que nos proporciona.

Tres fueron los maestros herreros que participaron en las obras: Tomás Blanco, al que se le pagaron cuarenta y cuatro pesos y cinco reales por varios trabajos sin especificar; Ventura Tapia, que cobró otros treinta y tres reales por varios trabajos; y Tomás Cárdenas, que recibió veintidos pesos y cinco reales por varios trabajos también sin determinar.

12. Cfr. TORERO GOMERO,C.: Opus cit. Páginas 444 a 447.

13. Ver apéndice documental, documento $n^{\circ} 1$.

14. A.G.I. Cuzco 21 y Lima 697. Testimonio del expediente promovido sobre la habilitación de la Casa y compra de muebles necesarios para la formación de la real Audiencia del Cuzco y sus oficinas.

15. A.G.I. Cuzco 21 y Lima 697. 
Un mayor número de carpinteros quedan reflejados en la relación de gastos. Estos se ocuparon de la realización de las ventanas, puertas, balaustradas, tarimados, puertas de rejas de madera de la cárcel, sillas y mesas.

Entre las anotaciones de pagos a los maestros carpinteros aparecen algunas sin determinar su trabajo, suponemos se trata de los artesanos que se dedicaron a los encargos menos artísticos. Son los siguientes: Pablo Alvarez, al que se le pagaron ciento ocho pesos por su trabajo; Tadeo Marquina, que cobró ciento cinco pesos; Miguel Morán, que recibió ochenta y un pesos; Manuel Villavisencio, que cobró sesenta y dos pesos; Pablo Monrroy, que percibió veinticuatro pesos; Francisco Carreño, que recibió treinta y nueve pesos y seis reales, y Jacinto Arias, al que se le pagaron veintiseis pesos y cuatro reales.

Al maestro carpintero Francisco Marquina, también vecino del Cuzco, se le pagaron noventa y nueve pesos por las once sillas de la Sala del Acuerdo. El inventario y descripción de la casa y muebles del Cabildo ${ }^{16}$ al que ya menos hecho mención, nos las describe " forradas con terciopelo carmesí, guarnesidadas con un galoncito de oro y tacheladas con clavason dorados con barníz morado y perfiladas con oro, y sus coronaciones igualemente doradas, y la del señor presidente se distingue en la coronación que tiene una palomita y su fleco de oro en el medio".

A Pablo Álvarez, maestro carpintero del que ya hemos reseñado un pago por varios trabajos sin especificar, se le encarga la restauración del púlpito de la Capilla de Santa Bárbara. Esta capilla, localizada en la casa "subiendo a la mano derecha", estaba sirviendo para a la Real Audiencia, y a ella se le adjudicó un púlpito de los bienes expropiados a los jesuítas, que el citado Pablo Alvarez recompuso.

El maestro hojalatero, Francisco Manzano, se encargó de colocar todas las vidrieras de las puertas y ventanas de la casa, por lo que se le pagaron cuarenta y siete pesos.

Los dos escudos con las armas reales colocados en los doseles de la Salas de Acuedo y de Justicia, fueron encargados al maestro bordador, Matias de León, por los que cobró ciento setenta y tres pesos y seis reales. Dichos escudos estaban bordados en hilo de oro, plata y seda con gran profusión de bramante.

La Sala de Justicia se adornó con un reloj de péndola que fue compuesto por el maestro relojero, Gregorio Rubí, por lo que se le pagaron veinticinco pesos. Este artista era maestro de relojería y platería, como así lo demuestra el contrato de aprendizaje del niño Marcos del Pezo, por el que el citado Gregorio Rubí se comprometía a enseñarle el oficio de platero ${ }^{17}$.

En el arco de arranque de la escalera que subía a las dependencias de la Real Audiencia, se colocó una lápida de bronce con la siguiente inscripción:

16. Ver apéndice documental, documento $n^{\circ} 1$. A.G.I. Cuzco 21 .

17. CORNEJO BOURONDE, J.: Arte Cuzqueño IV. REVISTA DEL ARCHIVO HISTORICO DEL CUZCO, n 5, 1954. Páginas 297 y 298. 


\begin{abstract}
"DE ORDEN DE S.M. EL SEÑOR DON CARLOS TERCERO, GOVERNANDO ESTOS REYNOS,COMO VIRREY EL EXCMO. SEÑOR DON TEODORO DE CROIX, SE HISO Y ESTABLECIO ESTA REAL AUDIENCIA, POR SU PRIMER REGENTE GOVERNADOR INTENDENTE DE ESTA CIUDAD Y SUS PROVINCIAS, EL SEÑOR DON JOSE PORTILLA Y GLAVEZ AÑO DE MIL SETECIENTOS OCHENTA Y OCHO".
\end{abstract}

Esta la realizó el maestro fundidor, Vicente Ribas, por la que percibió veinte pesos. En las dependencias de la Real Audiencia existían algunos lienzos decorando las distintas salas, así como algunas pinturas en tablas como las de un señor San José y un San Juan Nepomuceno que decoraban las puertas del oratorio.

Varios retratos decoraban la Sala de Justicia, entre ellos uno del rey Carlos IV, otro de la reina doña Luisa de Borbón, otro, de cuerpo entero, del regente José Portilla y uno, de medio cuerpo, del Marqués de la Sonora, don José Gálvez. De estos trabajos pictóricos sólo sabemos el autor del retrato del rey. Este fue el maestro pintor, José de Barrio, vecino de la ciudad del Cuzco, por el que se le pagaron treinta y cinco pesos. También sabemos quienes realizaron los escudos de armas y los trofeos que se veían en las distintas salas, se trata de los pintores Antonio Villegas, por los que recibió seis pesos, e Ignacio Chacón, al que se le pagaron ocho pesos y siete reales.

Los gastos dedicados a la pintura y dorado de las paredes ${ }^{18}$, puertas, ventanas y marcos también quedan reflejados en la documentación. Así, nos encontramos un pago al maestro dorador, Nicolás Cruz, de cuarenta y cuatro pesos.

De estas anotaciones lo más interesante es la referencia que se hace a dos mujeres, denominadas en la documentación como "maestras" y reconociéndolas como auténticas artistas independientes, desligadas de los habituales talleres familiares en los que trabajaban anónimanente. Se trata de la maestra pintora y doradora, Francisca Peso, que incluso se hacía acompañar por un aprendiz llamado Ignacio Rosalba; y de la maestra doradora, llamada Doña Clara la doradora. La primera realizó varios trabajos de pintura y dorado por los que cobró 200 pesos, mientras que la segunda se encargó del dorado de los marcos de los retratos.

\title{
2.c. Costes.
}

A pesar de los intentos por controlar los gastos de las obras y acondicionamiento de la Sede de la Audiencia, estos se dispararon. De los 4.000 pesos que estimó Mata LInares en su informe, se aumentó hasta 13.312 pesos y 6 reales, coste final de las mismas. De ellos unos 8.000 pesos se destinaron básicamente a las obras del

18. Las paredes se pintaron, en su mayoría, de verde y con bordes dorados, al igual que las puertas y las ventanas. 
edificio, mietras que los otros 5.000 pesos restantes fueron destinados al equipamiento de muebles y enseres.

Después de haber sido aprobada la relación de gastos tanto por el virrey don Francisco Gil como por la Junta Superior de Hacienda de Lima, se solicita la aprobación de la Corona. Para ello el virrey Gil remite una carta fechada en Lima a 16 de marzo de 1791, por la que solicita la aceptación de los citados gastos ${ }^{19}$.

Se resuelve en primera instancia, remitiéndola al Consejo con Real Orden de 29 de septiembre de 1791. El Consejo decide dar su opinión en favor de cargar el total de todos los gastos a la Audiencia del Cuzco, y el 5 de octubre del mismo año, por acuerdo envía el expediente a la Contaduría General y al fiscal, que no estimaron excesivos los gastos. Finalmente, son aprobados y tenidos a cuenta de la Real Hacienda.

\section{APENDICE DOCUMENTAL}

\section{Documento $n^{\circ} 1$}

Inventario y descripción del estado de la Casa del Cabildo tras su acondicionamiento para acoger a la sede de la Audiencia, fechado en la ciudad del Cuzco a 20 de enero de 1789, y mandado a realizar por el regente Portilla ante el escribano José Agustín Chacón Becerra. Documento incluido en el expediente sobre los gastos de las obras de la citada casa, que a su vez es documento anejo a una carta del virrey del Perú, Francisco Gil, fechada en Lima a 16 de marzo de 1791. A.G.I. Cuzco 21 y Lima 697.

" Inventario== En la ciudad del Cuzco, en viente dias del mes de septiembre de mil setecientos ochenta y nuebe años, El Señor don Jose Portilla y Galvez del Consejo de Su Magestad, Regente de esta real Audiencia, Gobernador Intendente desta Provincia y Partidosde su comprehension, en conseqüencia de lo mandado en el Auto que antecede, acistido de mi el presente escrivano de camara interino de esta dicha Real Audiencia, paso a hacer noticion, y prolijo Inventario de lo que contienen estas casas de la Real Audiencia, que antes de su execcion fueron propias del Cabildo y Ayuntamiento, en las que abitaba su corregidor la qual se hiso en la forma siguiente $==$ Dicha casa esta situada en la Plaza Principal nombrada del Regocijo, que hace frente a las Casas de la Moneda: su puerta de calle es alta de dos ojas, y ensima tiene las armas Reales,y de la Ciudad, al entrar en la mano derecha del saguan, hay una piesa que esta dedicada para la escribania de Camara, con una ventana que cae a dicha

19. A esta carta del virrey Gil, fechada en Lima a 16 de marzo de 1791 ( 2 folios), le acompañan:

- Testimonio sobre los gastos habidos para la casa y muebles de la Nueva Audiencia del Cuzco, (82 folios);

- Testimonio sobre la habilitación de la casa y compra de muebles necesarios para la formación de la Real Audiencia,

(30 folios).

Esta carta y su documentación aneja aparecen duplicadas en A.G.I. Cuzco 21 y Lima 697. 
Plaza, con sus rejas de hierro y puertas, no tiene mueble alguno, pues el escribano propio debera poner todo lo que necesitare. La puerta de su entrada es nueva de dos ojas pintada al oleo de berde:su cerradura, y demas corriente. En la mano isquierda de dicho Saguan esta otra piesa, que es la Chancilleria Real:tiene un docel de damasco carmesi, con su fleco de lana del mismo color, y en medio un escudo mayor de las Armas Reales pintado en lienso, de una vara de largo, y su marco dorado;el Docel tiene tres paños, y como dos varas delargo: Hay tres mesas forradas en baqueta, con clavason de metal amarillo, y sus flecos de lana carmesi de vara y media de largo: la una para escribir, ysobre la otra un forcula o prensa para sellar. Una escalera de tablas, con quatro andamuos: una alacena archivera con quatro puertas enrrejadas, y una barillade hierro de asegurar dichas puertas: tiene una alacena pequeña con chapa y llave y puerta de dos ojas forrada en papel de China pintado, que sirve para guardar los Sellos Reales, que estan en su caxa. dentro de esta misma alacena esta una almoada de terciopelo carmesi, con franjas vellotas, y cordones de oro, y su paño de cubierta de tesu, con su fluequesito de oro. tambien tiene una ventana que cae a la citada Plaza con enrrejado de hierro, y su Puerta principal de dos ojas, con chapa y llave y pintada de berde==

Sigue al Patio la primera puerta a mano derecha, que es antigua y sirve para el manejo, y uso interior del señor Regente de esta Real Audiencia, que como Governador Intendente abita en ella== Sigue otra que todavia estas sin destino== Sigue la Sala nueba del Ayuntamiento, con su ante Sala, Archivo, y otras piesas, con varios adornos y muebles, que unos son de los que tenia la Sala antigua, yotros executados de nuebo, para cuya obra nueba, fue necesario mudar el corral y caballerisas, que correspondian a estas piesas de Ayuntamiento, y se formaron unos Arcos de cal y ladrillo, y un corredor alto para facilitar y no impedir la comunicacion interior. Despues de estasigue otra piesa que sirvio de Sala de visita de carcel, y confesiones, la qual tiene comunicacion por una nueba Puerta a dicha Carcel. En la dicha Sala en su textera se forma el tribunal, sobre un estrado de tarima con quatro gradas, todo esterado, y con una alfombra, y en el mismo testero hay un Docel con su espaldar de damasco carmesi, que cubre casi hasta el suelo. En el medio esta otro lienzo de escudo mayor de Armas Reales, con su Docel igual al de la Chansilleria. Tiene una mesa delante con su ferliz de damasco carmesi, con flueco de seda del mismo color forrada en listadillo. A esta piesa sigue la carcel unica, que tiene esta ciudad para hombres, con tres puertas de reja de madera. $\mathrm{Al}$ frente de dicha carcel, hay un corredor alto, y bajo de arcos de piedra labrada, y balaustres de madera pintados de berde, que todo se hiso de nuebo. LLegando a la escalera que sube del primer arco, esta una lapida de bronce que tiene la siguiente descripcion: DE ORDEN DE SU MAGESTAD EL SEÑOR DON CARLOS TERCERO; GOVERNANDO ESTOS REYNOS; COMO VIRREY EL EXCELENTISIMO SEÑOR DON TEODORO DE CROIX, SE HISO Y ESTABLECIO ESTA REAL AUDIENCIA,POR SU PRIMER REGENTE GOVERNADOR INTENDENTE DE ESTA CIUDAD Y SUS PROVINCIAS, EL SEÑOR DON JOSE PORTILLA Y GLAVEZ AÑO DE MIL SETECIENTOS OCHENTA Y OCHO. Sobre dicha lapida ahy(hay) un Escudo grande de Armas reales de piedra de Berenguela, perfilada con oro; en el descanso deestaescalera estan pintadas las Armas Reales de escudo mayor, y subiendo a la mano derecha esta una puertecita para entrar a la capilla Real de Santa Barbara, que ha estado sirbiendo a la Real Audiencia, que tiene un pulpito, que se le adjudico de los bienes de los Exjesuitas. En esta escalera, hay dos Arcosnuebos: uno al principio, y otro al fin de ella: Su techo parece se hiso de nuebo, y parte de sus paredes. En dicho corredor principal, la primera puerta es de dos ojas de quatro varas de alto, y dos y quarta de ancho, 
con su postigo, cerrojo y cerradura, todas de color berde. Por ella se entra primero a la Sala de Procuradores, y es la siguiente $=$

$\mathrm{N}^{\circ} 1$ SALA DE PROCURADORES== Tiene quatro mesitas con sus respectivos caxoncitos para dichos, y sus asientos pegados a lapared en forma de escaños. A un lado de dicha Sala está una Alhacena que ocupa el Relator, con sus puertas de dos ojas de molduras y pintura verde y. perfiles de oro: dicha piesa es espaciosa;toda ella pintada y bien aseada y parece haverse hecho su techo nuevo, como tambien la pared, que corresponde al corredor, por haverse hecho su techo nuevo, como tambien la pared, que corresponde al corredor, por haverse estado cayendo la antigua. Tiene una mampara grande delante de la puerta del real Acuerdo de dos caras en que esta pintada la cesion del Escorial, y de su sagrario: Tiene dos ventanas que caen a la Plaza, con sus vidrieras de cristales, Una puerta principal de dos caras con sus molduras, pintadas de verde y dorado, que hace anteSala a la de Justicia, y en este con medio hay una puerta de igual clave para dicho corredor o baranda de la Plaza, con una ventana de cristales. Hay en esta anteSala una mesita y escañito llano para el secretario y relator. En el expresado corredor o baranda hay dos puertas con sus picaportes, que forman tres divisiones $=$

$N^{\circ} 2$ SALA DE JUSTICIA== Esta tiene once varas de largo y siete de ancho: Su puerta principal de molduras barnisada de verde, y perfilasa de oro: toda ella esterada y colgada con treinta y siete paños de papeles pintados. Asu textura esta un Dosel, que ocupa todo su ancho de terciopelo carmesi. El docel tiene en un canto galon de oro y flueco de lo mismo. En el medio de dicho espaldar esta su escudo mayor de las Armas Reales; bordado con ilo de oro, plata y ceda. El espaldon, y toda la colgadura tienen arriba y abajo molduras de madera de media caña doradas. El canape, que esta bajo de dicho Docel, es de quatro varas de largo con barniz colorado, y perfiles de oro, forrado con terciopelo carmesi, y un galoncito de oro y tachuelas doradas: tiene delante una mesa forrada con baqueta de larga de tres y una tercia varas con su faldon de terciopelocarmesi y guarnicion de la misma franxa de oro que cubre los tres lados de la mesa y por el frente cuelga sobre las gradas del trono. Ensima tiene quatro tomos de a folio en pergamino las leyes de partida, tres tomos de las leyes de Castilla, forrados igualmente en pergamino. Quatro tomos de leyes de Indias empastada, un tomo de Ordenanzas del peru y otro de Intendentes y por todo trece piezas. Una Campanilla del Plata con peso de quatro marcos seis onzas, dos tinteros de lo mismo con sus tapas y el interior de plomo, y ojalata: el uno con peso de cinco marcos, siete onzas y el otro con cinco marcos dos onzas, una salvadera con tres marcos seis onzas. Un oblearis con su tapa con cinco marcos una onza. El piso de dicha testera es entarimado con quatro gradas: tiene alfombras de seis varas de largo: de forma que las dos y media varas vajan las gradas u de ancho todo el de la Sala, y ambos costados de esta hay asientos de a dos tablas de largo para los abogadosque sirven igualmente de caxas con su grada, o estrado: al frente del tribunal esta un camape(canapé) forrado en baqueta con flueco de lana carmesi y tachuelasde metal dorado en el que se sientan el relator y escrivano de Camara, y delante una mesa forrada con igual baqueta, flueco y barniz y ensima tintero y salvadera de plomo. Tambien hay otro escaño grande de valaustres, tras de dicho camape que sirve de varanda y de asiento para procuradores. Hay una ventana bien grande con sus puertas con quatro ordenes de cristales, y ademas sus puertas de madera de varniz verde, perfil de oro y ensima una repisa imperial dorada con 
su cortinage de damasco, con su flueco de seda. Ygual repisa u cortina hay a la puerta de la entrada y al costado derecho hay una alacena grande con sus puertas igual en pintura y dorado con su chapa, llave, picaporte, y tinados; siguiente a la qual estacolocado el Relox de pendola Real, con diario segundero, minutero de campana, y su cajon. A los lados de la puerta hay dos alacenas, la una del escrivano de camara, y la otra del relator con varnis verde, y perfiles de oro; con sus chapas y llaves inglesas. Dos Plumeros Españoles al lado de dicha puerta principal: hay dos tablillas, la una de dias feriados y la otra de oficios. Frente de esta Real Sala, mediando la de Procuradores, esta la de Real Acuerdo==

$\mathrm{N}^{\circ} 3$ SALA DE REAL ACUERDO= Tiene de largo diez y media varas, y de ancho siete varas: su puerta principal grande de molduras varnisada de verde, y perfil de oro, esterada toda ella colgada con treinta y ocho paños de papeles ajardinados de color de aurora, iguales a los de la Real Sala de Justicia, y en ambos estremos sus molduras de media caña doradas: a su testura esta un docel grande forrado en terciopelo carmesi guarnecido de galon de oro, y flueco de los mismo: su espalda que cubre todo el testero de igual terciopelo carmesi, y alredor su moldura de media caña dorada, y en el medio estan las Armas reales en el Escudo mayor vordadas con hilo de oro, plata y seda. Al lado derecho esta el retrato de nuestro soberano el señor don Carlos quarto, con su moldura de media caña. Al izquierdo esta otro igual retrato de la Reyna nuestra Señora Doña Luisa de Borbon. Tambien hay un retrato del excelentisimo señor Ministro de Indias Marques de la Sonora don Jose Galves. Otro del Excelentisimo Señor don Teodoro de Croix del medio cuerpo y una vara de largo, y otro de cuerpo entero de mas de dos varas del señor don Jose Portilla Regente Governador Intendente etcetera. Frente dicha Sala su entarimado de seis varas de largo y quatro de ancho, cuvierto de una alfombra de siete y media varas de largo y seis varas de ancho: alrededor estan once sillas forradas con terciopelo carmesi, guarnecidas con un galoncito de oro y tachueladas con clavason dorada con varnis morado, y perfiladas con oro, y sus coronaciones igualmente doradas, y la del señor presidente se distingue en la coronacion que tiene una Palomita, y su flueco de oro en el medio. Hay dos mesas forradas en Baqueta con sus faldones hasta el suelo de terciopelo carmesi, y por la clavason guarnesidas con su galoncito, y tachuelas doradas: ensima esta una campanilla de plata con peso de tres marcos cinco onzas: un tintero de plata, con su interior de plomo y oja de lata, y su tapadera con peso de cinco marcosdos onzas: su salvadera con peso de tres marcos dos y media onzas; y asi en estas piezas como en las de la Real Sala de Justicia esta gravado un escudo de Armas Reales. Al frente del Tribunal hay otra mesa de vara y media de largo y tres quartas de ancho forrada en baqueta, con su flueco, y tachuelas doradas, con su Banca sin espaldar, tambien forrada en Baqueta, e iagual clavason que sirve para el Secretario de Camara: sobre esta mesa hay un tintero y salvadera de plomo. Tiene una puerta igual a la principal que cae al Balcon, o Galeria de la Plaza con otras puertas de cristal su repisa y cortina Ymperial de Damasco, y en la puerta principal hay igual cortina y repisa tambien. Tambien hay un armario o escaparate embutido en la pared y resguardo a Cal y Ladrillo que sirve de Archivo del real Acuerdo con las Puertas de igual moldura que las demas; pintura verde, perfiles de oro con tres Chapas, o cerraduras: Yglesas y sus picaportes. Tambien ay a los costados y estremos de dicha Sala tres Bancas sin espaldar de largo de dos y tercia varas, y del ancho de una tabla con forro de baqueta y flueco de lana carnesi, y tachuelas doradas, seis almoadas de terciopelo carmesi con franjas de oro y seis vellotas alos cantos de hilo de oro, y un citiar o paño de mesa de terciopelo de dos varas y 
media forrado con tafetan amarillo, con guarniciones de franja de oro y flueco de los mismo que sirve para las funciones de la Yglesia. Hay otra puerta de molduras verde, y perfilada de oro que corresponde a un quarto de desahogo para los señores ministros y es trancito para la Capilla de Santa Barbara. En dicho quarto hay una ventana con valaustres. Todo este transitosegun parece se hizo de nuevo asi el techo como el suelo==

ORATORIO== Esta es una piesa que tiene correspondencia con la Real Sala de Justicia con su puerta corriente chapa y llaves. Sigue otra puerta con vidrieras que cae al balcon que se hizo de nuevo que tambien esta pintado de verde, y cae a la misma Plaza. Tiene otra puerta que corresponde a las havitaciones interiores. Esta toda esta piesa esterada con una alfombra y colgada de papeles ajardinados. En la frente hay una puerta grande de dos ojas pintadas para un lado de verde, y perfilada de oro, y por el otro estan pintados al oleo un Señor San José y San Juan Nepomuceno que es la que forma el Oratorio, en el que hay quatro Blandocitos de plata con peso de diez y siete marcos y una lamina con su marco de plata y su vidrieras de la efigie de Nuestra Señora de la Interiora, y un Santo Cristo en bulto con peana, su frontal de guadamesi, el Altar es una mesa con sus caxones, chapas y llaves y forrado dicho oratorio en papel ajardinado. Tambien tiene una Alfombra de cinco y tres quartas varas de largo y de ancho dos tres quartas varas=== Para el Servicio del Altar de este oratorio provicionalmente se han aplicado varias casullas, singules, ara, mantel y otros paramentos que estan en las reales Caxas por via de pocito como especies sequestradas a los Vilinsurgentes (viles e insurgentes) Jose Gabriel y Diego Tupaamano (Túpac Amaru), las que se custodiaron en una mesa dorada con caxones y chapas y llaves que en el ante oratorio esta colocada, y se individualisaran expecificamente quando se recivan. Ygualmente al un lado, digo estremo de este anteoratorio hay una division o tavique con su puerta, y adentro hay un cajon con su vidriado para desahogo de los señores, y siguiente a esta piesa hay un atrio en forma de obalo con sus gradas de piedra, y puerta que franquea la comunicacion interior a las Casas de vivienda, y morada del señor Regente Presidente Governador Intendente de esta Provincia, las que estan repartidas y en las que en tiempo antiguos siempre han havitado los corregidores que han sido de esta Ciudad, con lo qual se concluyo esta Notacion e Inbentario quedando a cargo de los porteros los muebles de las piezas que corren de su cuenta y tienen en su poder las llaves y tambien a la del Señor Chanciller Registrador de lo tocante a su oficina, y lo firmo su señoria de que certifico: siendo testigos don Marcos Rivera, don Blas Marin Porteros deesta real Audiencia, y don Pedro Davalos presentes=

$\begin{array}{ll}\text { Jose Portilla }== & \text { Blas Marin de Velasco== } \\ \text { Marcos Rivera=== } & \text { Don Jose Agustin Chacon y Becerra." }\end{array}$




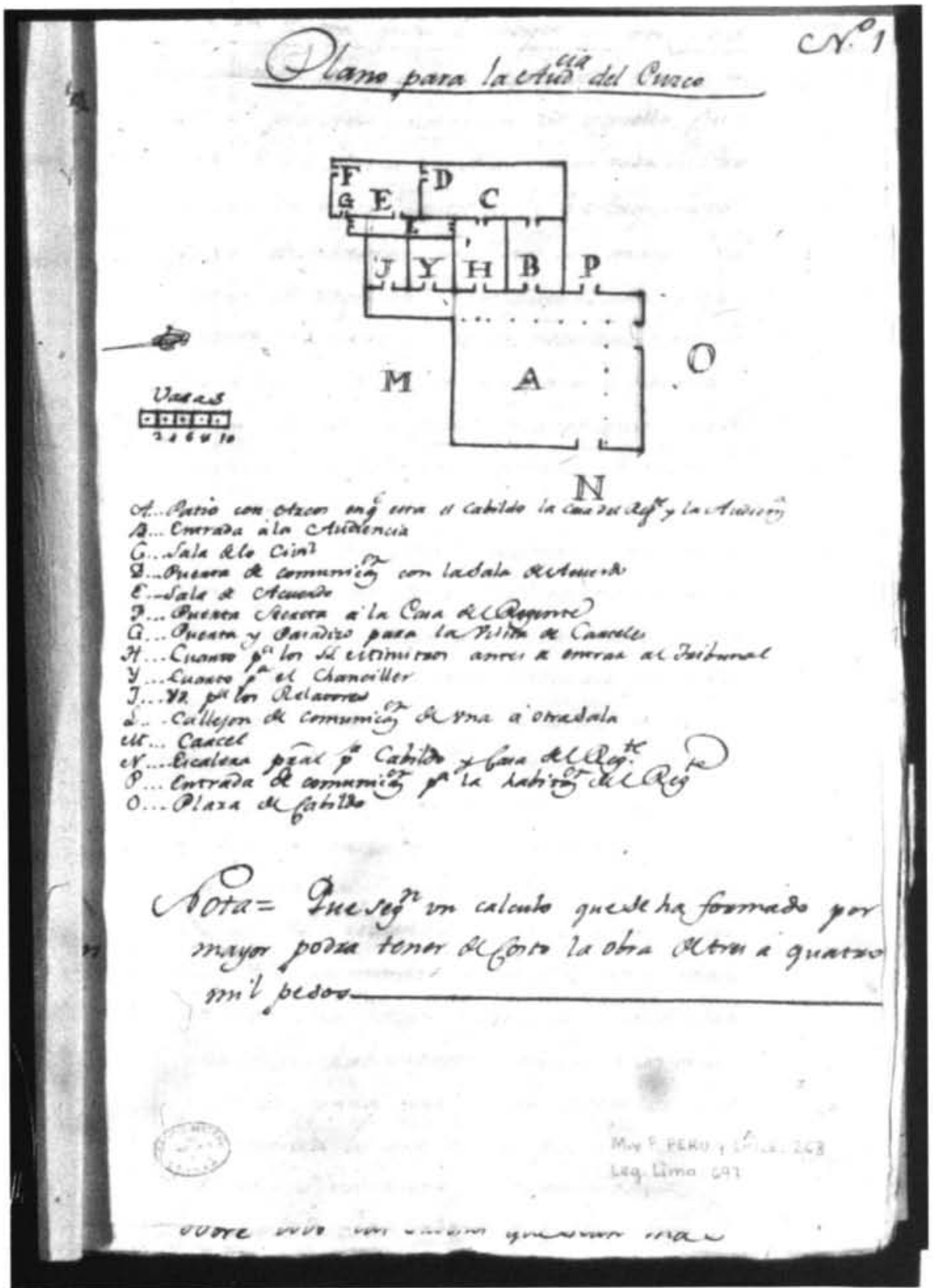

Fig. 1.

Plano de la Casa de la Audiencia, ajuntado por Mata Linares a su informe, sugiriendo la futura distribución de ésta. A.G.I. Mapas y Planos, Perú y Chile, 268, 268 bis. 


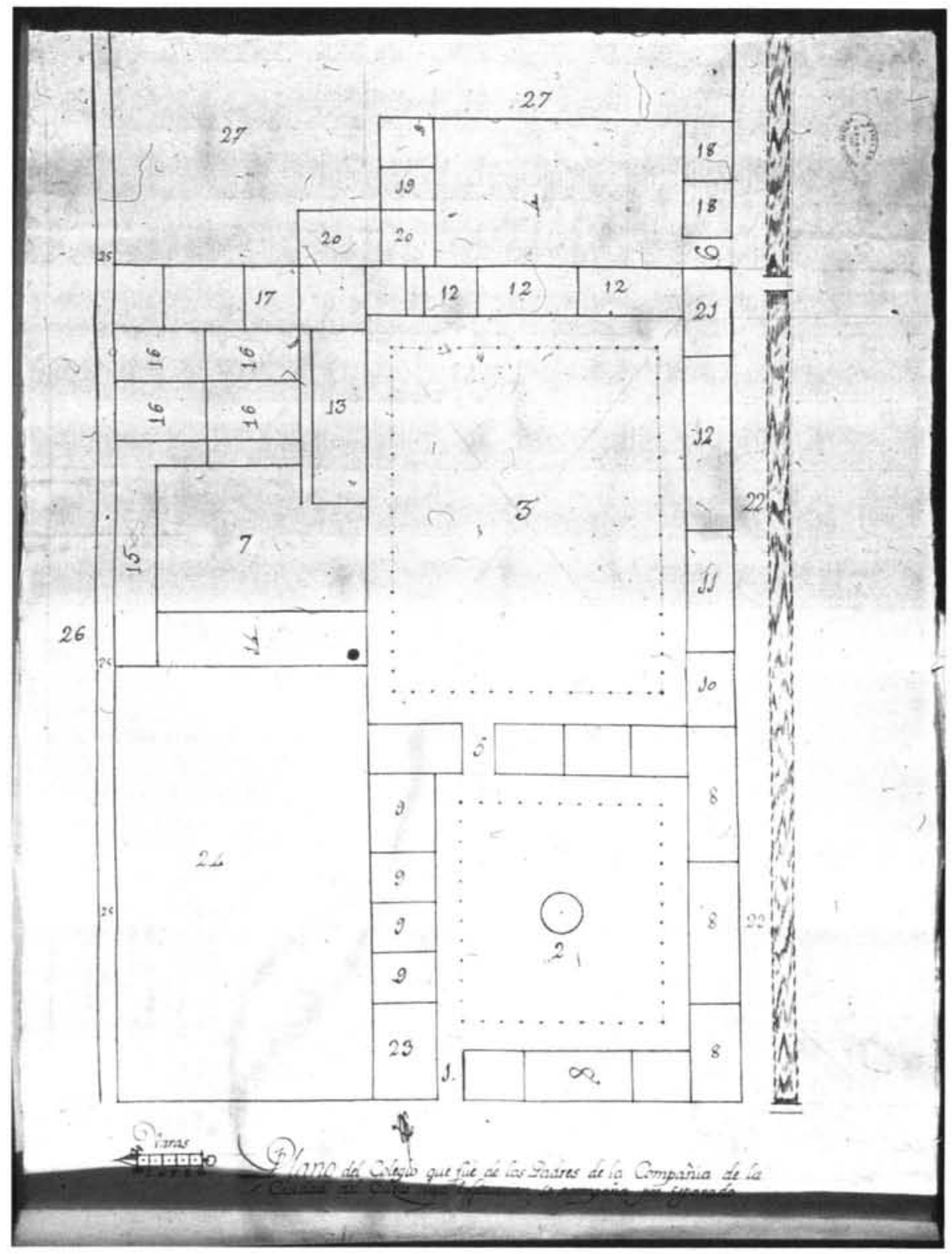

Fig. 2.

Plano de la Casa de la Compañía de Jesús, edificio también propuesto como sede de la Audiencia, anejo al informe de Ignacio Delgado. A.G.I. Mapas y Planos, Perú y Chile, 267, 267bis. 